\title{
Shear Thinning and Local Melting of Colloidal Crystals
}

\author{
Roel P. A. Dullens ${ }^{1}$ and Clemens Bechinger ${ }^{2,3}$ \\ ${ }^{1}$ Department of Chemistry, Physical and Theoretical Chemistry Laboratory, University of Oxford, \\ South Parks Road, Oxford OX1 3QZ, United Kingdom \\ 2. Physikalisches Institut, Universität Stuttgart, Pfaffenwaldring 57, 70569 Stuttgart, Germany \\ ${ }^{3}$ Max-Planck-Institute for Intelligent Systems, Heisenbergstrasse 3, 70569 Stuttgart, Germany
}

(Received 18 April 2011; published 19 September 2011)

\begin{abstract}
Phenomena such as shear thinning and thickening, occurring when complex materials are exposed to external forces, are generally believed to be closely connected to changes in the microstructure. Here, we establish a direct and quantitative relation between shear thinning in a colloidal crystal and the surface area of the locally melted region by dragging a probe particle through the crystal using optical tweezing. We show that shear thinning originates from the nonlinear dependence of the locally melted surface area on the drag velocity. Our observations provide unprecedented quantitative evidence for the intimate relation between mechanical properties and underlying changes in microscopic structure.
\end{abstract}

DOI: 10.1103/PhysRevLett.107.138301

PACS numbers: 82.70.Dd, 64.70.dj, 83.50.-v, 83.60.Df

The response of materials to external stresses and strains is of central importance for many applications in science and technology $[1,2]$. At macroscopic length scales the microstructure of many materials and complex fluids is often neglected and continuum models are applied to describe their flow behavior [1-3]. As a consequence of progressive miniaturization, the effect of the microscopic structure on mechanical properties becomes increasingly significant, which has invoked the development of micromechanical models [3-6]. Also in complex fluids there are many suggestions that the rearrangement of the local structure is the basis for behavior like shear thickening and thinning [1,2,7-10]. The key here is the simultaneous characterization of the external forces and changes in the microstructure. We achieve this using active microrheology of colloidal crystals, which enables us to directly connect shear thinning to local melting.

A two-dimensional, hexagonal colloidal crystal of melamine spheres of radius $R_{c}=1.5 \mu \mathrm{m}$ in water is deformed using a probe particle trapped in an optical tweezer. The lattice spacing $a$ and number density $\rho$ are $3.5 \mu \mathrm{m}$ and $0.087 \mu \mathrm{m}^{-2}$ respectively, and the size of singledomain crystallites is typically larger than $250 \times$ $250 \mu \mathrm{m}^{2}$. Adding a very small amount (less than one probe particle per $\sim 3000$ small particles) of large polystyrene probe particles $\left(R_{p}=7.75 \mu \mathrm{m}\right)$ to the suspension results in a crystal with built-in probe particles as shown in Fig. 1(a). The probe particle is trapped by an acoustooptical-deflection controlled laser tweezer [11,12] and subsequently moved typically $\sim 100 \mu \mathrm{m}$ through the colloidal crystal at drag velocities $v$ varying from 0.05 to $4 \mu \mathrm{m} / \mathrm{s}$. Particle positions are monitored in real-space and time using digital video microscopy [13].

Driving the probe particle by optical tweezing can lead to three different modes: constant force, constant velocity or a mixed mode $[9,14]$. At constant force the probe can move around obstacles while at constant velocity the particle will largely resist lateral motions thus leading to significantly larger deformations of the material. To address the deformation of the crystal by the probe we used a stiff optical trap to suppress the diffusive motion of the probe particle in the trap during drag, and hence, establish a constant velocity regime [12]. The optical trapping potential was confirmed to be parabolic $U_{\text {trap }}=-\frac{k}{2} \Delta r^{2}$, with a spring constant $k=3.1 \times 10^{-7} \mathrm{~N} \mathrm{~m}^{-1}$ [Fig. 1(e)] [12].

Figure 1(a)-1(c) shows three snapshots of a crystal through which a probe particle is being dragged from left to right at a velocity of $0.25 \mu \mathrm{m} / \mathrm{s}$. As is clearly inferred from the disordered area behind the probe particle, the drag leads to the formation of numerous defects resulting in local melting [9,15-18]. Although the colloidal crystal is slightly compressed in front of the probe particle, the crystal remains remarkably intact in this region. This is due to the softness of the interparticle potential. Furthermore, the original crystal orientation is completely recovered behind the probe as recrystallization is templated by the crystal surrounding the disordered area [Fig. 1(c)]. The drag force can be directly determined from the displacement of the probe particle relative to the optical trap: $\Delta r \equiv\left|\vec{r}_{\text {probe }}-\vec{r}_{\text {trap }}\right|$. In Fig. 1(d) the displacement is shown as a function of time for a drag velocity of $0.25 \mu \mathrm{m} / \mathrm{s}$. Initially, the displacement increases steeply after which a steady state displacement $\Delta r_{s s}$ is reached [horizontal line in Fig. 1(d)]. The fluctuations around $\Delta r_{s s}$ are due the intermittent nature of the probe's motion through the crystal: pushing the encountered particles away and subsequently "hopping" from site to site $[9,17,18]$. The data presented here all correspond to the steady state regime.

The dependence of the steady state displacement—and hence the drag force $\left(F=k \Delta r_{s s}\right)$ - on the drag speed is presented in Fig. 1(f) and can be divided into two regimes. 

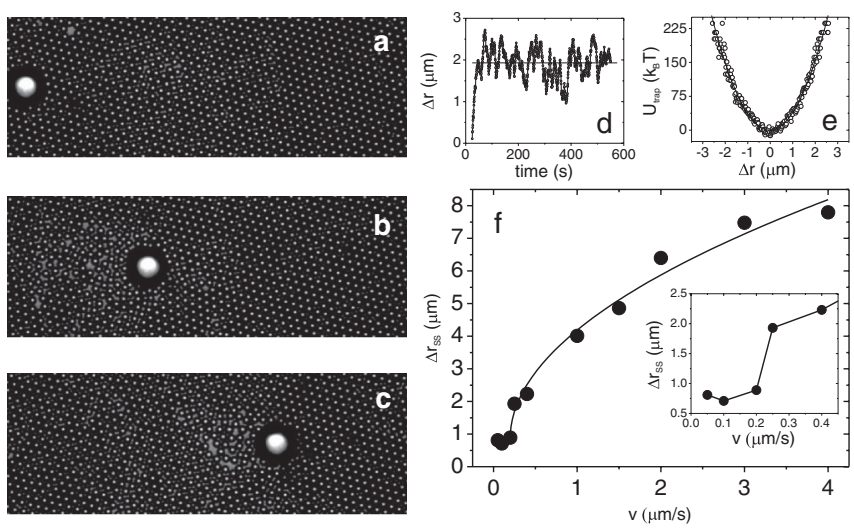

FIG. 1. (a)-(c) Snapshots $\left(170 \times 60 \mu \mathrm{m}^{2}\right)$ of the system for $v=0.25 \mu \mathrm{m} / \mathrm{s}$ at (a) 25, (b) 250 , and (c) $500 \mathrm{~s}$. Note that the drag was started at $t_{0}=25 \mathrm{~s}$. (d) The radial displacement of the probe particle from the center of the trap $\Delta r$ as a function of the time. The horizontal line represents the steady state displacement $\Delta r_{s s}$. (e) The trapping potential for the probe particle: $U_{\text {trap }}=-\frac{k}{2} \Delta r^{2}$ with the spring constant $k=$ $3.1 \times 10^{-7} \mathrm{~N} \mathrm{~m}^{-1}$. (f) $\Delta r_{s s}$ as a function of the drag velocity. The solid line is a power-law fit $F \sim\left(v-v_{c}\right)^{\alpha}$ with $\alpha=0.51$. The inset shows $\Delta r_{s s}$ for small drag velocities.

In the limit of very small drag velocities the steady state displacement only very slowly depends on the drag velocity [inset Fig. 1(f)]. The fact that the drag force jumps to a finite value for a nonzero drag velocity points to the existence of a finite yield stress [19-21]. At the yield point the crystal starts to deform plastically, which is the onset of melting. The force corresponding to yielding is $0.26 \mathrm{pN}$, which is very similar to values obtained in (3D) dense hard sphere fluids and glasses [22-24]. Converting the force to a stress is not straightforward as the area over which this force is applied is poorly defined. To obtain an order of magnitude for the yield stress we estimate this area to be on the order of $\left(2 R_{c}\right)^{2} \approx 10 \mu \mathrm{m}^{2}$. This leads to a yield stress of roughly $0.02 \mathrm{~Pa}$, which is comparable to earlier macroscopic rheology experiments on crystals of charged colloids [19].

At a drag velocity of around $0.20 \mu \mathrm{m} / \mathrm{s}$ the displacement starts to significantly increase with increasing drag velocity [inset Fig. 1(f)]. For drag velocities below this characteristic velocity $v_{c}$, the crystal recrystallizes faster than it is locally disrupted; hence, the probe particle is essentially dragged through the crystal without melting it. As a consequence, the injected power $F v$ (energy per unit time) is completely dissipated by friction below $v_{c}$. For $v>v_{c}$ the system does not recrystallize on the time scale of drag. Therefore, an increasing part of the crystal is locally melted upon increasing drag velocity, which leads to the development of a fluidlike area behind the probe particle. In this regime, the displacement-and hence the drag force-grows sublinearly with the drag velocity. Using a power-law fit we find that $F \sim\left(v-v_{c}\right)^{\alpha}$ with $\alpha$ close to $1 / 2$. This implies "velocity or shear thinning" similar to that observed in microrheology studies of dense hard sphere systems $[9,22,23,25]$ and bulk rheology of colloidal crystals $[19,20]$. Moreover, the $v^{1 / 2}$ behavior has been reported to be directly related to a change in the structure factor, suggesting a change in the local structure $[7,8]$. In our experiments, the change in the local structure is readily quantified by analyzing the observed local melting of the crystal, which allows us to directly elucidate the connection between shear thinning and the microstructural rearrangements.

The local structure is characterized by computing the defect density relative to the position of the probe particle averaged over the steady state [12]. The defect densities are shown for four different drag speeds in Fig. 2(a). The color scale represents the fraction of time (in the steady state) the system has been fluidlike. The area of the fluidlike region behind the probe particle clearly increases with drag speed. The-to a first approximation - "triangularlike" shape of the defect area is the result of local melting and recrystallization: without recrystallization the defect area would be rectangular. The time required to recrystallize from rectangular to triangularlike is defined as the recrystallization time and it is given by $t_{r}=L_{y} / v_{r}$, where $L_{y}$ is the perpendicular defect length [see Fig. 2(b)] and $v_{r}$ the recrystallization speed. The shape of the defect area furthermore suggests that it recrystallizes laterally as shown in Fig. 2(b), consistent with the observation that the particles constitute the same area before and after local melting.

Using a threshold of 0.5 for the fraction of time that the system has been fluidlike to distinguish between crystal and fluid, we determined the surface area of the observed defect area as a function of the drag velocity [Fig. 2(c)]. This area consists of an area behind, $A_{b}$, and in front of, $A_{f}$, the probe. Comparing the total area to the defect area in front of the probe, shows that the defect area behind the probe dominates the observed total defect area, and hence, that the structure in front of the probe remains intact. Below the characteristic velocity $v_{c}$ the total defect area does not depend on the drag velocity, which yields a "static defect area" $A_{0} \approx 340 \mu \mathrm{m}^{2}$ corresponding to an effective static defect probe radius $L_{0} \approx 10 \mu \mathrm{m}$. This is consistent with a "frustrated" fluid layer of roughly one small particle diameter around the probe as reported for crystals containing large spherical impurities [26].

Above $v_{c}$, the power injected into the crystal is mainly dissipated through distortion of the colloidal crystalresulting in local melting - rather than dissipation by friction as is the case below $v_{c}$. This is evident from the fact that below $v_{c}$ the increase of $\Delta r_{s s}$ with $v$ is negligible compared to its increase above $v_{c}$ [Fig. 1(f)]. As a result, the drag force can be quantitatively related to the local melting for $v>v_{c}$. The number of melted particles per unit time equals $\rho A_{\text {melt }} / t_{r}$, where the total melted area $A_{\text {melt }}$ is given by $\left(2 A_{b}-A_{0} / 2\right)+\left(A_{f}-A_{0} / 2\right)=2 A_{b}+$ $A_{f}-A_{0}$. The multiplication factor two accounts for the 

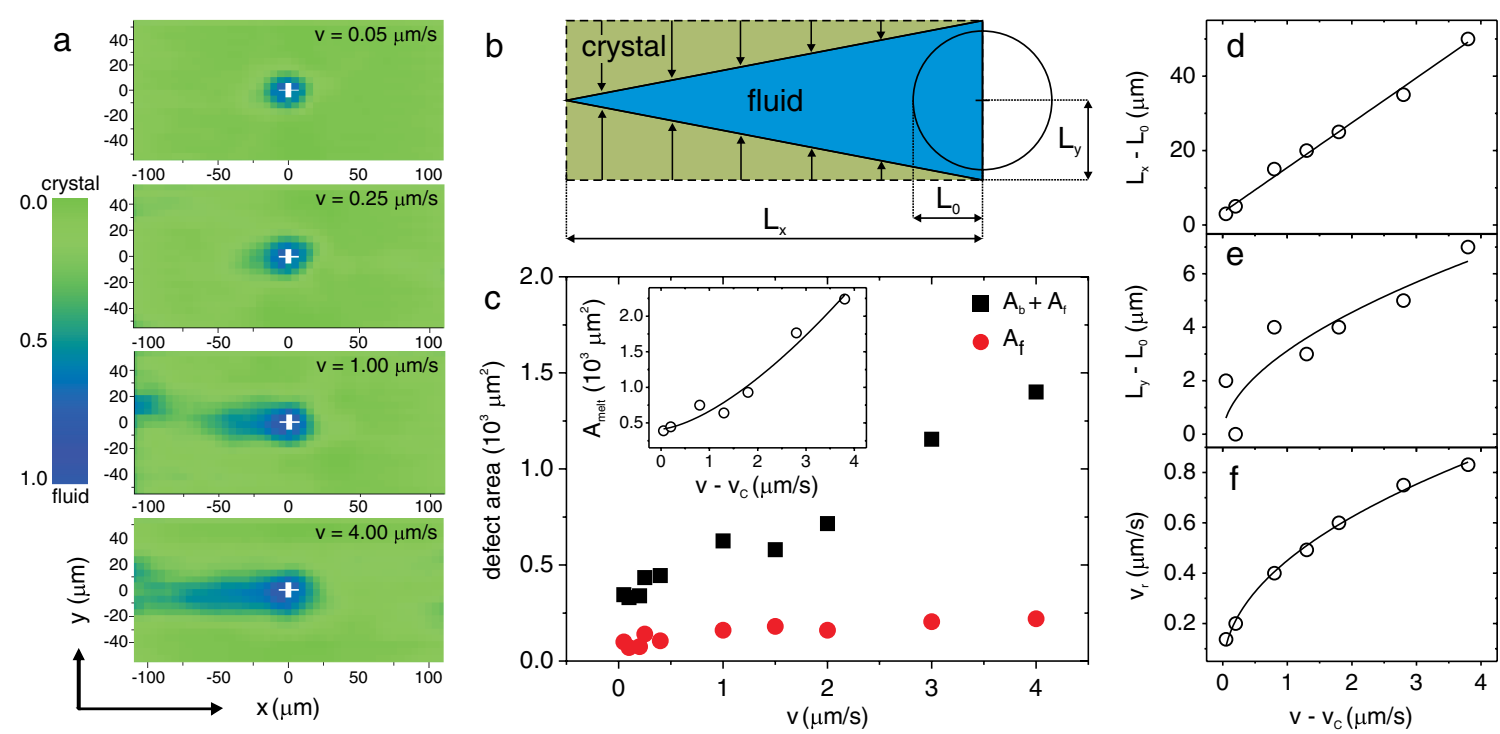

FIG. 2 (color online). (a) Defect densities for four different drag velocities. The color scale is the fraction of time in the steady state that the system has been fluidlike: green (blue) corresponds to crystalline (fluidlike) areas. (b) Schematic of the recrystallization process in which the particles are moving in laterally. The parallel and perpendicular defect lengths $L_{x}$ and $L_{y}$, and the effective static defect radius of the probe $L_{0}$ are indicated. (c) The observed total defect area (squares) and the defect area in front (dots) of the probe particle as a function of $v$. The inset shows the melted area $A_{\text {melt }}$ as a function of $v-v_{c}$. The solid line is a power-law fit $A_{\text {melt }} \sim\left(v-v_{c}\right)^{\alpha}$ with $\alpha=1.51$. (d) $L_{x}-L_{0}$ as a function of $v-v_{c}$ with a linear fit to the data. (d) $L_{y}-L_{0}$ as a function of $v-v_{c}$ with a power-law fit $L_{y} \sim\left(v-v_{c}\right)^{\alpha}$ with $\alpha=0.55$. (f) The recrystallization velocity as a function of $v-v_{c}$. The solid line is a power-law fit $v_{r} \sim\left(v-v_{c}\right)^{\alpha}$ with $\alpha=0.47$.

fact that the total melted area behind the probe is rectangular, in contrast to the observed shape which is triangularlike due to recrystallization (Fig. 2). By defining the melting energy per particle $U_{m}$, the injected power is directly related to $A_{\text {melt }}$ for $v>v_{c}$ through

$$
\left(F-F_{0}\right) v=U_{m} \rho A_{\text {melt }} / t_{r},
$$

where $F_{0}$ is the "yield force." Importantly, Eq. (1) provides a direct quantitative relation between shear thinning and the microstructure in terms of local melting. The observed shear thinning behavior thus directly originates from the nonlinear dependence of the melted surface area on the drag velocity.

For drag velocities larger than $v_{c}$ the drag force is-to leading order-proportional to $v^{1 / 2}$. In this regime, the total melted surface area $A_{\text {melt }}$ is expected to be proportional to $v^{3 / 2}$, which is confirmed by the power-law fit to the data in the inset in Fig. 2(c). To further corroborate this, we note that $A_{\text {melt }} / t_{r}$ is approximately given by $2 L_{y} L_{x} / t_{r}=2 L_{y} v$, where $L_{x}$ and $L_{y}$ are the parallel and perpendicular defect lengths [Fig. 2(b)]. From Eq. (1) it then follows that $L_{y}$ has the same drag velocity dependence as the drag force $F$. Figure 2(e) confirms that $L_{y} \sim v^{\alpha}$ with $\alpha$ close to $1 / 2$. Consistently, the parallel defect length $L_{x}$ is found to be proportional to $v$ [Fig. 2(d)]. Interestingly, this suggests that the recrystallization velocity $v_{r}$ depends on the drag velocity $v$ : in the time $t_{r}$ the crystal has healed a distance $L_{y}=v_{r} t_{r}$, and the probe has moved a distance
$L_{x}=v t_{r}$. Note that this implies that $t_{r}$ is constant as $L_{x} \sim v$. The recrystallization velocity is then given by $v_{r}=\left(L_{y} / L_{x}\right) v$. Because $L_{x} \sim v$ and $L_{y} \sim v^{\alpha}$ the recrystallization velocity $v_{r}$ should be proportional to $v^{\alpha}$ with $\alpha$ close to $1 / 2$, which is indeed observed in Fig. 2(f). We suspect that this reflects the increasing number density mismatch between the crystal and the melted region at higher drag speeds, which leads to a higher driving force for recrystallization and hence a larger $v_{r}$. We also expect the hydrodynamic flow generated by probe particle to affect the recrystallization, especially at higher drag velocities.

The established relation between the drag force and the local structure also directly leads to the melting energy per particle. Plotting the injected power as a function of the melted area for $v>v_{c}$ indeed yields linear dependence as shown in Fig. 3 and from the slope we find $U_{m}=152 \pm$ $12 k_{B} T$. This rather high value reflects the nonequilibrium nature of the drag-induced melting in which all "bonds" are broken. In this limit, $U_{m}$ may be approximated as $z U_{i j}(a) / 2$ [27], where $z$ is the coordination number and $U_{i j}(a)$ the pair interaction at a distance $a$. This leads to $U_{i j}(a) \approx 50 k_{B} T$, which is common for charged colloidal systems at high number densities [28].

From the melting energy per particle we can additionally estimate Young's modulus $E$, which is defined as $E=$ $\sigma / \gamma$, where $\sigma$ is the stress and $\gamma$ the strain. Dividing the stress by the number density $\rho$ yields an energy per particle. Subsequently, applying the modified 2D 


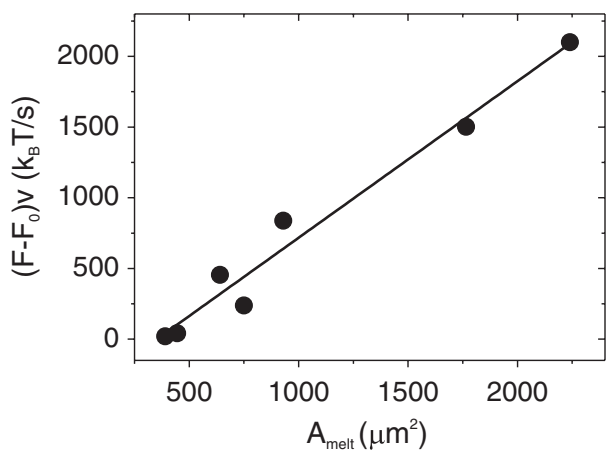

FIG. 3. The power as a function of the total melted area $A_{\text {melt }}$ for $v>v_{c}$. The linear fit to the data yields the melting energy per particle using Eq. (1).

Lindemann's criterion for the yield strain $\gamma=\gamma_{\text {yield }} \approx$ 0.033 [29] relates the melting energy per particle to the Young's modulus

$$
\frac{\sigma}{\rho}=U_{m}=\frac{E \gamma_{\text {yield }}}{\rho} \rightarrow E=\frac{U_{m} \rho}{\gamma_{\text {yield }}} .
$$

Interestingly, from our local probe experiments we find $E \approx 560 k_{B} T / a_{0}^{2}$, consistent with measurements on extended bulk 2D colloidal crystals [30,31]. The good agreement of our value for $E$ with macroscopic results suggests that this bulk concept is still valid even at microscopic length scales.

In conclusion, we have used laser tweezers to manipulate a two-dimensional colloidal crystal at microscopic length scales by dragging a probe particle through the crystal. Simultaneously measuring the drag force and monitoring the change in the microscopic structure has enabled us to establish a quantitative relation between the observed shear thinning and the local melting of the crystal. The fact that the surface area of the locally melted region grows as $v^{3 / 2}$ directly leads to drag force being proportional to $v^{1 / 2}$. Based on this relation, values for the melting energy per particle and the Young's modulus of the crystal were found, which suggests that these macroscopic concepts are still valid at length scales comparable to the particle size. We believe that our experiments confirm the direct connection between flow properties like shear thinning and the microstructure. It would therefore be very exciting to extend these measurements to other complex materials such as glasses, ceramic suspensions, and biological systems, though quantifying the change in the microstructure in noncrystalline systems might be more cumbersome.

We thank Christopher Hertlein, Jens Harting, Rudolf Weeber, Eric Weeks, Paul Chaikin, Daniel Bonn, and Jan Dhont for fruitful discussions, and Susan Perkin and Michael Juniper for critically reading the manuscript. RPAD acknowledges the Alexander von Humboldt Foundation for financial support.
[1] R. G. Larson, The Structure and Rheology of Complex Fluids (Oxford University Press, Oxford, 1999).

[2] J. F. Morris, Rheol. Acta 48, 909 (2009).

[3] S. Li and G. Wang, Introduction to Micromechanics and Nanomechanics (World Scientific Publishing Co., Ltd., Singapore, 2008).

[4] S. Ogata, J. Li, and S. Yip, Science 298, 807 (2002).

[5] F. Célarié, S. Prades, D. Bonamy, L. Ferrero, E. Bouchaud, C. Guillot, and C. Marlière, Phys. Rev. Lett. 90, 075504 (2003).

[6] W. K. Lui, S. Jun, and D. Qian, J. Comput. Theor. Nanosci. 5, 970 (2008).

[7] J. K. G. Dhont, J. C. Van der Werff, and C. G. De Kruif, Physica (Amsterdam) 160A, 195 (1989).

[8] J.C. van der Werff, C. G. de Kruif, and J. K. G. Dhont, Physica (Amsterdam) 160A, 205 (1989).

[9] I. C. Carpen and J. F. Brady, J. Rheol. 49, 1483 (2005).

[10] N. J. Wagner and J. F. Brady, Phys. Today 62, 27 (2009).

[11] D. Babic, C. Schmitt, I. Poberaj, and C. Bechinger, Europhys. Lett. 67, 158 (2004).

[12] See Supplemental Material at http://link.aps.org/ supplemental/10.1103/PhysRevLett.107.138301 for detailed information about the colloidal system, optical tweezing, and the defect density calculation.

[13] J. C. Crocker and D. G. Grier, J. Colloid Interface Sci. 179, 298 (1996).

[14] T. M. Squires and J.F. Brady, Phys. Fluids 17, 073101 (2005).

[15] J. A. Weiss, D. W. Oxtoby, and D. G. Grier, J. Chem. Phys. 103, 1180 (1995).

[16] D. L. J. Vossen, Ph.D. thesis, Utrecht University, 2004.

[17] C. Reichhardt and C. J. Olson Reichhardt, Phys. Rev. Lett. 92, 108301 (2004).

[18] C. Reichhardt and C. J. Olson Reichhardt, Phys. Rev. E 69, 041405 (2004).

[19] S. Mitaku, T. Ohtsuki, and K. Okano, Jpn. J. Appl. Phys. 19, 439 (1980).

[20] H. M. Lindsay and P. M. Chaikin, J. Phys. Colloques 46, C3-269 (1985).

[21] A. Pertsinidis and X. S. Ling, New J. Phys. 7, 33 (2005).

[22] P. Habdas, D. Schaar, A.C. Levitt, and E. R. Weeks, Europhys. Lett. 67, 477 (2004).

[23] A. Meyer, A. Marshall, B. G. Bush, and E. M. Furst, J. Rheol. 50, 77 (2006).

[24] L. G. Wilson, Ph.D. thesis, University of Edinburgh, 2007.

[25] L. G. Wilson, A. W. Harrison, A. B. Schofield, J. Arlt, and W. C. K. Poon, J. Phys. Chem. B 113, 3806 (2009).

[26] V.W. A. de Villeneueve, L. Derendorp, D. Verboekend, E. C. M. Vermolen, W. K. Kegel, H. N. W. Lekkerkerker, and R. P. A. Dullens, Soft Matter 5, 2448 (2009).

[27] D. A. McQuarrie and J.D. Simon, Molecular Thermodynamics (University Science Books, Sausalito, 1999).

[28] J. A. Weiss, A. E. Larsen, and D. G. Grier, J. Chem. Phys. 109, 8659 (1998).

[29] K. Zahn, R. Lenke, and G. Maret, Phys. Rev. Lett. 82, 2721 (1999).

[30] K. Zahn, A. Wille, G. Maret, S. Sengupta, and P. Nielaba, Phys. Rev. Lett. 90, 155506 (2003).

[31] K. Q. Zhang and X. Y. Liu, Langmuir 25, 5432 (2009). 